\title{
2015-2016 Reviewers-Consultants 2015-2016
}

The following individuals have graciously provided reviews for the Canadian Journal of Experimental Psychology during the period of November 2015 to November 2016. We thank them for their thoughtful and timely assistance.

De novembre 2015 à novembre 2016, les personnes suivantes ont offert leur collaboration spéciale dans l'évaluation de manuscrits pour la Revue canadienne de psychologie expérimentale. Nous les remercions de leur aide précieuse.

$\begin{array}{ll}\text { Herve Abdi } & \text { Peter Dixon } \\ \text { James S. Adelman } & \text { Sidney D'Mello } \\ \text { Naseem Al-Aidroos } & \text { Jason Droll } \\ \text { Philip Allen } & \text { Chad Dube } \\ \text { Ben Amsel } & \\ \text { Mark Ashcraft } & \text { Anita Eerland } \\ & \text { James T. Enns } \\ \text { Marie-Laure Barbier } & \text { Zachary Estes } \\ \text { Anthony Barnhart } & \text { Thomas Faulkenberry } \\ \text { Raoul Bell } & \text { Jonathan M. Fawcett } \\ \text { Dan Bernstein } & \text { Susanne Ferber } \\ \text { Derek Besner } & \text { Tom Foulsham } \\ \text { Adam Biggs } & \text { John Franchak } \\ \text { Marcus Bindemann } & \text { Megan Freeth } \\ \text { Walter Bischof } & \\ \text { Benjamin Boller } & \text { Giovanni Galfano } \\ \text { Ty Boyer } & \text { Arthur Glenberg } \\ \text { Benoit Brisson } & \text { Hayward Godwin } \\ \text { James Broadway } & \text { Robert Gordon } \\ \text { Timothy Brown } & \text { Davood Gozli } \\ \text { Neil Bruce } & \\ \text { Julie Bugg } & \text { Matthias Hartmann } \\ \text { Thomas Busey } & \text { Heather Henderson } \\ \text { Serge Caparos } & \text { William E. Hockley } \\ \text { Alan D. Castel } & \text { William Horrey } \\ \text { Luigi Castelli } & \text { Michael Hout } \\ \text { Craig Chapman } & \text { Carol Hudon } \\ \text { Neil Charness } & \text { Mike Humphreys } \\ \text { Yalin Chen } & \\ \text { Jim Clark } & \text { Roxane Itier } \\ \text { Louise Connell } & \text { Jason Ivanoff } \\ \text { Denis Cousineau } & \\ \text { Greg Cox } & \text { Rachael Jack } \\ \text { Kirsten Dalrymple } & \text { Debra Jared } \\ \text { Polly Dalton } & \text { Michelle Jarick } \\ \text { Zoltan Dienes } & \text { Brendan Johns } \\ & \text { Aaron P. Johnson } \\ \text { Bianca Jovanovic } \\ \end{array}$

\author{
Hilary Kalagher \\ Mike Kalish \\ Pentti Kanerva \\ Justine T. Kao \\ John Kennedy \\ Raymond Klein \\ Derek Koehler \\ Naomi Koerner \\ Gustav Kuhn \\ Peter Kwantes
}

Kaitlin Laidlaw

Jason P. Leboe-McGowan

Jean-Francois Lepage

Daniel Levin

Jason Lodge

Hugues Lortie-Forgues

Carol Madden-Lombardi

Catherine Manning

Hadas Marciano

Beat Meier

Douglas Mewhort

Mariko Nakayama

Ian Neath

Andy Ng

Brendan Norman

Erika Nurmsoo

Antje Nuthmann

Bettina Olk

Chris Oriet

Steven Prime

Daniel Richardson

Tobias Richter
Steven Roodenrys

Jean-Yves Roussey

Eric Ruthruff

Meghan Salomon

Christopher Sears

Judith M. Shedden

Scott Sinnett

Daniel Smith

Grayden Solman

Michael Spivey

Matthew Stainer

Biljana Stevanovski

Jennifer A. Stolz

David Strayer

Karl Szpunar

Laurence Taconnat

Gerry Tehan

David Thomson

Sébastien Tremblay

Chi-Shing Tse

Joseph Tzelgov

Wieske Van Zoest

Michèle Venet

Paul Verhaeghen

Troy Visser

Julia Vogt

Emily Ward

Scott Watter

Tim Welsh

Chris Westbury

Jeremy M. Wolfe

Lee Wurm

Motonori Yamaguchi 